\title{
Avaliação da maturação em crianças e jovens
}

\author{
Maturation assessment in children and adolescents
}

\author{
Dionizio Mendes Ramos Filho \\ Gustavo C. Lopes \\ Astrogildo V. Oliveira-Júnior*
}

\section{Resumo}

A avaliação da maturação é um aspecto importante da prática desportiva por parte de crianças e adolescentes. Existem diferentes métodos de avaliação em crianças e adolescentes, cada um com pontos fortes e fracos. Nas primeiras duas décadas de vida, a criança e o adolescente vivenciam três processos interativos: crescimento, maturação e desenvolvimento. O primeiro processo refere-se ao tamanho e proporções físicas; o segundo refere-se ao aprimoramento das funções esquelética, reprodutora, somática, neuroendócrina e neuromuscular. O terceiro processo refere-se ao desenvolvimento cognitivo, emocional, social, motor e moral. O presente texto apresenta uma revisão dos métodos de avaliação da maturação de crianças e adolescentes, descrevendo as características principais das diferentes técnicas, vantagens e desvantagens de cada uma delas. A maturação dos diferentes órgãos e sistemas ocorre em diferentes ritmos e momentos, levando-nos a concluir que a avaliação do

Revista HUPE, Rio de Janeiro, 2013;12(4):38-46 doi:10.12957/rhupe.2013.8711 estado de maturidade biológica varia com o sistema corporal considerado. Sendo assim, a eficiência de uma determinada estratégia está diretamente associada a sua capacidade de identificar os diferentes estágios de maturação biológica. Os sistemas mais comumente utilizados para avaliação da maturação são os sistemas esquelético, reprodutivo (sexual) e somático. A maturação dental (erupção e calcificação) é ocasionalmente utilizada, mas tende a se comportar independentemente dos outros três sistemas. Do ponto de vista hormonal a maturação também sofre grande influência dessas substâncias e também deve ser considerada. A determinação da maturidade esquelética, através da identificação da idade óssea, parece constituir-se no método que reúne mais vantagens na determinação do estágio maturacional de jovens, mesmo durante o período peripuberal.

Descritores: Determinação da idade pelo esqueleto; Determinação da idade pelos dentes; Crescimento e desenvolvimento; Saúde.

"Endereço para correspondência: Laboratório de Atividade Física e Promoção da Saúde, IEFD, UERJ. Rua São Francisco Xavier, 524, bloco F, $8^{\circ}$ andar, sala 8.121 Rio de Janeiro, RJ, Brasil. CEP: 20550-900. Telefone: 5521 2334-0495 E-mail: astrogildo.vojr@gmail.com 


\section{Abstract}

Maturation assessment is an important issue within sports practice by children and adolescents. In the first two decades of life, children and adolescents experience three interactive processes: growth, maturation and development. The first process refers to physical size and proportions; the second refers to skeletal, reproductive, somatic, neuroendocrine and neuromuscular functions; the third refers to cognitive, emotional, social, motor and moral issues. This text aimed to review the methods to assess the maturation of children and adolescents, describing their main characteristics, advantages and disadvantages. The maturation of the various organ systems occurs at different rates and times, leading us to conclude that the assessment of the state of biological maturity varies within the body system taken into consideration. The efficiency of a given strategy is directly linked to its ability to identify the different stages of sexual maturation. Systems most commonly used for evaluation of maturation are reproductive (sex) and somatic. Dental maturation (calcification and eruptions) is occasionally used, but tends to behave independently of the other three systems. Hormonal maturation influences all these aspects and should also be considered. However, skeletal maturity (bone age) seems to gather more advantages in determining the maturational stage of youth compared to the others, even if they are in peripuberal period.

Keywords: Age determination by skeleton; Age determination by teeth; Growth and development; Health.

\section{Introdução}

Nas primeiras duas décadas de vida, a criança e o adolescente vivenciam três processos interativos: crescimento, maturação e desenvolvimento. O primeiro processo refere-se ao tamanho, às proporções, ao físico, à composição e ao sistêmico; o segundo referese ao aprimoramento das funções esquelética, reprodutora, somática, neuroendócrina e neuromuscular; o terceiro processo refere-se ao desenvolvimento cognitivo, emocional, social, motor e moral. ${ }^{1}$ Este artigo se preocupa primordialmente com o segundo processo, qual seja a maturação.

Frequentemente confundido com crescimento e mais difícil de definir, o termo maturação é muitas vezes descrito como o processo de se tornar maduro, ou o progresso até o estado maduro. ${ }^{2}$ No entanto, um conceito mais amplo é apresentado por Bogin, ${ }^{3}$ para quem a maturação seria uma progressão de alterações quantitativas e qualitativas que conduzem de um estado indiferenciado ou imaturo a um estado altamente organizado, especializado ou maturo.

O ponto final da maturação, no contexto do crescimento, é o alcance da fase adulta, quando o indivíduo atinge a maturidade funcional, que no contexto biológico, implica na habilidade de procriar com sucesso e promover a descendência, a qual por si mesma procriará com sucesso. ${ }^{4}$ Assim, a maturidade sexual é a capacidade funcional reprodutiva plena, a maturidade esquelética é a completa ossificação do esqueleto adulto e a maturidade morfológica é o alcance das dimensões corporais de adulto.

De forma ainda mais ampla, a maturação é vista como um processo biológico contínuo e dinâmico, que se inicia na concepção e termina na morte. É pontuado por alterações visíveis na estatura, composição corporal e características sexuais secundárias, que culminam na transição da fase pré-reprodutiva para a reprodutiva do ciclo de vida humano. ${ }^{5} \mathrm{O}$ ritmo desta transição é variável e tem substanciais implicações sociais e biológicas. Além do genótipo, fatores exógenos ambientais, sociais e étnicos (clima, estação do ano, altitude, nutrição, nível socioeconômico, número de filhos na família e outros) também exercem considerável interferência sobre a maturação biológica. ${ }^{2}$

A maioria dos estudos epidemiológicos utiliza a idade cronológica como delimitador temporal. O início da vida escolar, as categorias esportivas e mesmo o crescimento 
normalmente são equivocadamente associados à idade cronológica, uma vez que esta, por não considerar o ritmo ou o momento biológico, não é um bom indicador da maturidade biológica. ${ }^{3}$ Os indicadores da idade de desenvolvimento são mais informativos sobre as características dos jovens em comparação com a idade cronológica. ${ }^{6}$ Jovens de diferentes estágios maturacionais são frequentemente encontrados em um mesmo grupo de treinamento ou categoria esportiva, o que pode favorecer aos mais adiantados no processo de desenvolvimento biológico, além de desmotivar outros mais tardios. ${ }^{7}$

Posto isso, o objetivo do presente artigo é revisar os principais métodos empregados para a avaliação da maturidade biológica, destacando-se suas vantagens, desvantagens e potencial aplicação a situações específicas.

\section{Maturidade sexual}

A avaliação da maturação sexual baseia-se na idade de aparecimento e evolução das características sexuais primárias e secundárias. As características sexuais primárias são aquelas diretamente envolvidas com a reprodução: desenvolvimento dos ovários, útero e vulva nas meninas; e desenvolvimento dos testículos, próstata e produção de esperma nos meninos. ${ }^{8}$ As características sexuais secundárias (CSS), associadas com o dimorfismo externo, são: desenvolvimento das mamas (DM) e idade da menarca (IM) nas meninas; desenvolvimento do pênis (DP) e dos pelos faciais e mudanças na voz nos meninos; e desenvolvimento dos pelos púbicos (PP) em ambos os sexos. Essas características são geralmente avaliadas de acordo com os cinco pontos da escala descrita por Tanner. ${ }^{9}$ As características sexuais secundárias refletem este importante sistema biológico e estão intimamente relacionadas com o eixo hormonal ${ }^{3}$ e com um importante período do desenvolvimento humano - a puberdade.

Frequentemente, os estágios de maturidade sexual são equivocadamente identificados como estágios de Tanner (menção ao responsável pela sua popularização), quando deveriam ser classificados como estágios de maturidade sexual, com a nomeação de características específicas avaliadas (mamas, pelos púbicos ou genitais). Os primeiros pesquisadores a estabelecerem os estágios de maturidade de características sexuais secundárias foram Reynolds e Wines, em 1948 e 1951, respectivamente. Os estágios de cada característica não são nem equivalentes nem intercambiáveis. $\mathrm{O}$ estágio 1 de cada característica indica o estado pré-púbere (ausência de desenvolvimento) enquanto o estágio 2 denota o início evidente do desenvolvimento de cada característica, marcando a transição para a puberdade. Os estágios 3 e 4 marcam o progresso na maturação e o estágio 5 indica o estado maduro (adulto). ${ }^{9}$

Os primeiros sinais aparentes da maturação sexual nas meninas são o desenvolvimento inicial das mamas, que ocorre em média por volta dos 10 anos, e nos meninos o aumento dos testículos e do pênis, por volta dos 11 anos. Em ambos os sexos, o sinal seguinte é o desenvolvimento dos pelos pubianos. ${ }^{1}$ Vários processos ocorrem durante este período (puberdade) que tipicamente dura 3-4 anos. ${ }^{10}$

A identificação dos estágios das características sexuais secundárias era inicialmente realizada por um médico, por observação individual no exame clínico. ${ }^{11} \mathrm{O}$ controle de qualidade (reprodutibilidade intra e inter-observadores) deve ser considerado. Nesse contexto, pode-se acreditar que a reprodutibilidade entre avaliadores experientes seja geralmente boa, com cerca de $80 \%$ de concordância na atribuição dos estágios, ${ }^{1}$ enquanto entre avaliadores sem experiência prévia as percentagens de concordância são baixas, na ordem de 40\%. ${ }^{1-3}$ Em ambientes não médicos, a autoavaliação com a utilização de fotografias ou desenhos, vem sendo mais utilizada. Esta deve ser realizada em ambiente privativo, numa sala tranquila e equipada com espelho, utilizando-se fotografias de boa qualidade dos estágios e descrições simplificadas, além de estar o jovem, preferencialmente acompanhado por seu responsável. É importante 
que o avaliador seja do mesmo sexo do avaliado para evitar distorções na classificação.

Por outro lado, aspectos desfavoráveis da avaliação da maturidade sexual através da observação dos CSS são relatados por alguns autores: a dificuldade de aplicabilidade na ausência de um médico especializado; as exigências específicas do local onde será feita a avaliação e os constrangimentos criados ao avaliado. ${ }^{10}$ Neste aspecto, pode haver hesitação de pais, organizações esportivas e comitês de ética em permitir a avaliação clínica dos caracteres sexuais secundários, devido a preocupações com a privacidade individual. Outras dificuldades apontadas remetem ao fato de as fotografias serem em preto-branco e os estágios serem arbitrários e discretos. ${ }^{12}$ Ao considerar as alterações ocorridas durante a puberdade, esta técnica restringe a avaliação aos períodos próximos da puberdade, não diferenciando outros períodos mais distantes da puberdade.

A idade da menarca (a primeira menstruação) é o indicador de maturidade sexual mais comumente empregado em meninas. ${ }^{1}$ Segundo Beunen e colaboradores, ${ }^{13}$ ela pode ser observada de três diferentes maneiras, de acordo com o tipo de informação desejada: prospectivamente (estudo longitudinal), interrogando-se a mesma menina sobre a ocorrência da menarca em intervalos regulares de 3 a 6 meses; retrospectivamente, interrogando-se meninas ou mulheres que já tenham vivenciado a menarca, perguntando-lhes quando a mesma ocorreu; e status quo, questionando grandes amostras de meninas de aproximadamente 9 a 16 anos sobre a menarca (pré ou pós-menarca). Os dois primeiros métodos fornecem a idade individual da menarca, enquanto o método do status quo proporciona uma estimativa da idade da menarca de uma amostra populacional.

$O$ equivalente no desenvolvimento masculino à menarca das meninas é a espermarca ou oigarca, segundo Duarte ${ }^{14}$ definida como a primeira ejaculação, frequentemente um evento involuntário que ocorre durante o sono.
Por ocorrer nestas condições, é uma medida impraticável em estudos epidemiológicos. Entretanto, o início da espermatúria, medida em amostras da primeira urina coletada de manhã cedo por 10 dias, prediz melhor o estágio clínico de puberdade do que a idade cronológica, podendo ser útil em estudos que envolvam a identificação da puberdade masculina. ${ }^{15} \mathrm{~A}$ espermatúria é um evento comum e regular nas fases inicial e média da puberdade, podendo ocorrer antes de qualquer outro sinal da puberdade. ${ }^{15}$ Quando avaliada repetidamente, é um procedimento sensível, eticamente aceitável e não invasivo de determinar o início da atividade espermatogênica em indivíduos púberes. A idade da espermarca é definida pela idade na qual a espermatúria pode ser identificada em $50 \%$ da população. ${ }^{15}$

\section{Maturidade esquelética ou óssea}

A maturação do esqueleto é amplamente reconhecida como o melhor indicador isolado do estado de maturidade. A idade óssea tem sido o indicador mais comumente usado nos estudos sobre crescimento e desenvolvimento, sendo considerada como um registro verdadeiro da idade biológica. Todas as crianças iniciam com um esqueleto de cartilagem e progridem até um esqueleto totalmente ossificado, adulto. No caso dos ossos tubulares (ossos longos e curtos), a maturidade é atingida quando ocorre a completa fusão das epífises com suas diáfises correspondentes; no caso dos ossos com formato irregular, a maturidade é definida pela morfologia adulta. ${ }^{3}$

A maturidade esquelética apresenta vantagens como um indicador da maturidade biológica: fornece uma estimativa razoavelmente precisa e confiável, é aplicável durante todo o período de maturação pós-natal e reflete a maturação de um importante sistema biológico contínuo, ${ }^{3}$ para além de, juntamente com a estatura numa certa idade, permitir predizer a estatura em adulto. ${ }^{1}$

Além de definir o momento maturacional, 
a idade óssea pode ser utilizada para definir o ritmo, quando dela se subtrai a idade cronológica. Com isso, podem-se classificar os jovens em síncronos, atrasados ou adiantados. Quando a diferença se mantém entre - 1 e +1 eles são classificados em síncronos, atrasados quando ela é maior que - 1 , e adiantados quando maior que $+1 .{ }^{16}$

\section{Método Greulich-Pyle (GP)}

O método mais utilizado de avaliação da maturidade esquelética é o de Greulich e Pyle, ${ }^{17}$ inclusive pela radiologia clínica brasileira. Sucessor do primeiro guia prático de avaliação da maturidade óssea por radiografia, idealizado por Wingate Todd, em 1937, o atlas de Greulich e Pyle, desenvolvido com crianças de famílias americanas de classe socioeconômica alta, ${ }^{17}$ é ainda muito utilizado, principalmente na avaliação clínica. Desde o primeiro guia prático, a avaliação é feita na mão e punho esquerdos. Greulich e Pyle ${ }^{17}$ sugeriram que a utilização de uma das mãos estaria associada a uma menor exposição à radiação e a um menor custo. Já a escolha da mão e punho esquerdos dever-se-ia a uma determinação do Acordo Internacional para Unificação das Medidas Antropométricas em indivíduos vivos, delineada na Conferência de Antropologistas Físicos, em 1906 e 1912, no Mônaco e em Geneva, respectivamente. Outro aspecto citado, que pode ter sido considerado, é o fato de haver mais indivíduos destros que canhotos nas populações em geral, o que diminuiria a possibilidade de haver mais mãos esquerdas mutiladas ou lesionadas para serem utilizadas nas avaliações.

Para determinar a maturidade óssea, deve-se inicialmente procurar no atlas a figura da radiografia correspondente ao sexo e idade cronológica do indivíduo que estamos estudando e verificar nas radiografias adjacentes (mais velha e mais nova) qual dos três filmes mais se assemelha superficialmente à radiografia a ser avaliada. Considerando-se o padrão de desenvolvimento para a idade, a ausência ou presença de alguns centros de ossificação carpais ou epifisiais, assim como a forma de determinados ossos pode fornecer pistas úteis quanto ao desenvolvimento ósseo.

\section{Método Tanner-Whitehouse (TW)}

A técnica apresentada por TannerWhitehouse encontra-se no seu segundo estágio de aprimoramento, sendo uma sucessão do método proposto por Greulich e Pyle. ${ }^{17}$ Ela se baseia na noção de maturidade fisiológica primeiramente proposta por Franz Boas, da Universidade de Berlim e Columbia. Como existe uma ordem invariável de acontecimentos relativamente ao desenvolvimento ósseo preceito para todas as técnicas que se utilizam da ossificação do esqueleto como método de avaliação da idade óssea, esta técnica fundamenta-se no desenvolvimento dos centros de ossificação de alguns ossos para obtenção de um valor que resulta do somatório dos seus diferentes graus de ossificação. Em 1962, Tanner, Whitehouse e Healy propuseram uma nova metodologia de avaliação da maturidade óssea (TW1), com um constructo matemático mais sólido. Cada osso da mão e do punho era observado separadamente e registrava-se um dos oito ou nove estágios possíveis. Em 1975, foi publicada uma revisão desta metodologia (TW2), modificando os valores atribuídos a cada estágio. Atribuía-se maturidades diferenciadas para os ossos carpais, rádio, ulna e ossos curtos, incluindo-se uma diferenciação por sexo. Com isso, permitia-se a estimativa da estatura adulta. Essa revisão foi realizada sobre dados da mesma amostra utilizada anteriormente, de 3.000 meninas e meninos britânicos. $\mathrm{Na}$ versão mais recente o TW $3^{18}$ considera apenas o escore RUS (rádio, ulna, metacarpos e falanges). Os sete ossos do carpo (escore carpal) não são mais incluídos e a idade esquelética passou a ser determinada por apenas 13 ossos. A amostra foi ampliada, constituindo-se de crianças britânicas, belgas, italianas, espanholas, argentinas, norte-americanas e japonesas. $\mathrm{O}$ método é considerado como sendo o mais 
objetivo. $^{18}$

$\mathrm{Na}$ leitura do filme identifica-se a quantidade de centros de ossificação presentes, o nível de ossificação apresentado pelos ossos e o número de fusões epifisárias ocorridas, segundo descrição do método TW3. ${ }^{18}$ Os ossos observados são: rádio, ulna, metacarpos (I, III e V), falanges proximais (I, III e V), falanges médias (III e V) e falanges distais (I, III e V). As classificações variam com a progressão do estágio de desenvolvimento, de A (estágio inicial) a I (estágio final - maduro) para a maioria dos ossos, com exceção da ulna, do osso grande (capitale), do pisiforme (triqueal), do semilunar (lunate), do escafoide e do trapezoide, que possuem a classificação $\mathrm{H}$ como seu estágio final de maturação. Se nenhuma característica estiver presente no osso, é assumida a classificação $\mathrm{A}$.

$\mathrm{Na}$ análise das radiografias deve-se considerar que o filme é a representação bidimensional de uma estrutura tridimensional. Este fato produz, pela sobreposição de duas superfícies penetradas pelos raios $\mathrm{X}$, um maior grau de branqueamento em comparação com o que se observa quando os raios $\mathrm{X}$ atravessam uma única superfície. Tanner e colaboradores ${ }^{18}$ recomendam a utilização de uma régua ou um paquímetro para fazer a comparação dos diâmetros ósseos, quando o critério determina que um osso deva ter a metade ou mais do tamanho de um outro.

\section{Maturidade dentária}

O desenvolvimento dentário também é utilizado como indicador maturacional. A maturidade dentária tem sido calculada tradicionalmente pelas idades de erupção dos dentes decíduos e/ou permanentes, o número de dentes presentes em certa idade cronológica, ou a idade na qual um número específico de dentes tenha aflorado. O rompimento da gengiva é frequente e erroneamente chamada de erupção, uma vez que representa apenas um estágio do processo contínuo de erupção dental ou de migração para alcançar o estágio de oclusão. $\mathrm{O}$ rompimento pode ser influenciado por ancilose, extração precoce ou tardia dos dentes decíduos, impacto e empurrão dos dentes permanentes. Entretanto, se o aparecimento é usado como critério de avaliação da idade dental, ele somente pode ser aplicado até a idade de 30 meses (conclusão da dentição decídua) e após a idade de seis anos (erupção do primeiro molar). ${ }^{19}$

Muitos estudos ${ }^{19}$ têm investigado a associação entre a emergência e a formação da raiz. A emergência visível geralmente ocorre quando a formação da raiz está 3/4 completada, entretanto variações consideráveis têm sido observadas. ${ }^{19}$ A erupção é só um evento no processo de calcificação dos dentes e possui limitado significado biológico. Além disso, os critérios para erupção dentária variam, por exemplo, da perfuração inicial da linha da gengiva até a completa erupção. ${ }^{3}$ A maturidade dental também é observada com fins forenses, buscando estimar a idade cronológica de indivíduos abaixo e acima dos 20 anos. Entretanto, a idade dental não pode ser utilizada para estimar a idade cronológica em indivíduos não maturados, sob pena de se atribuir à idade cronológica um indicador do desenvolvimento biológico.

A calcificação dental, como avaliada em radiografia, também promove uma indicação de estado de maturidade. Demirjian e colaboradores ${ }^{20}$ desenvolveram uma escala de maturidade dental, baseada nos princípios do método de Tanner-Whitehouse (TW1) para avaliação de idade esquelética. $O$ procedimento requer uma radiografia panorâmica dos sete dentes em um quadrante da boca (dois incisivos, o canino, dois pré-molares e os primeiro e segundo molares). Como no sistema de Tanner-Whitehouse, indicadores específicos de maturidade são identificados para cada dente, os estágios são classificados em uma escala de maturidade para cada dente, e os escores são subsequentemente somados para prover um escore global de maturidade dental. ${ }^{3}$ 


\section{Maturidade morfológica ou somática}

A avaliação do nível maturacional através da idade morfológica baseia-se no pressuposto de que as alterações da velocidade de crescimento estatural e de outras medidas lineares, tais como altura troncocefálica (sentado) e comprimento dos membros inferiores, apresentadas por um indivíduo ao longo do seu processo de crescimento refletem a sua evolução maturacional. Entretanto, uma vez que todos os indivíduos adultos não são iguais, o tamanho corporal isoladamente não é um indicador válido da maturidade biológica. ${ }^{3}$

O salto ou surto na velocidade de crescimento (SVC) indica um período da puberdade no qual a velocidade de crescimento aumenta e em que ocorre o pico na velocidade de crescimento em estatura (PVE). Nesse momento, no qual a velocidade de crescimento alcança o seu maior valor, representa-se a idade do pico da velocidade de crescimento (IPVC). Para Petersen e colaboradores, ${ }^{21}$ o PVC é especialmente relevante como uma medida do momento puberal.

Durante o crescimento extrauterino a estatura e a massa corporal aumentam gradualmente, existindo momentos em que a velocidade de crescimento em estatura ou dos segmentos corporais aumenta substancialmente. O primeiro momento, menos intenso, é chamado salto da velocidade de crescimento pré-púbere ou crescimento intermediário (6 a 8 anos). A sua identificação, após ele ter ocorrido, pode ser difícil, uma vez que os aumentos na taxa de crescimento são frequentemente pequenos para serem clinicamente perceptíveis, especialmente nas meninas. O segundo momento ocorre durante a puberdade, podendo a velocidade de crescimento alcançar 7-10 cm/ano, com ligeira desvantagem para as meninas. ${ }^{9} \mathrm{O}$ segundo aumento na velocidade de crescimento, mais intenso e tardio nos meninos, dá início ao salto de crescimento púbere, primeiro em estatura e depois em massa corporal. ${ }^{3} \mathrm{O}$ PVC ocorre quando o crescimento linear atinge aproximadamente $92 \%$ da estatura em adultos.

A utilização de medidas corporais implica a obtenção de dados antropométricos individuais ao longo de um período de um ano ou mais. ${ }^{22} \mathrm{Um}$ acompanhamento longitudinal é necessário, ${ }^{1}$ sendo desejável um intervalo máximo de seis meses para o melhor acompanhamento das alterações. A avaliação de maturidade somática baseada nos parâmetros da curva de crescimento é limitada ao período da puberdade, e são considerados apenas um ou dois eventos biológicos. ${ }^{3}$ Quando se consegue obter um valor preditivo da estatura final em adulto de crianças ou adolescentes a percentagem da estatura adulta alcançada em uma determinada idade pode ser usada como indicador de maturidade. ${ }^{3}$

Os indicadores de maturação somática mais utilizados são: ${ }^{3}$ a) idade de início do aumento da velocidade de crescimento púbere (primeiro ponto de inflexão na curva de crescimento púbere - SVC); b) idade em que se atinge a velocidade máxima de crescimento púbere (segunda inflexão na curva de crescimento

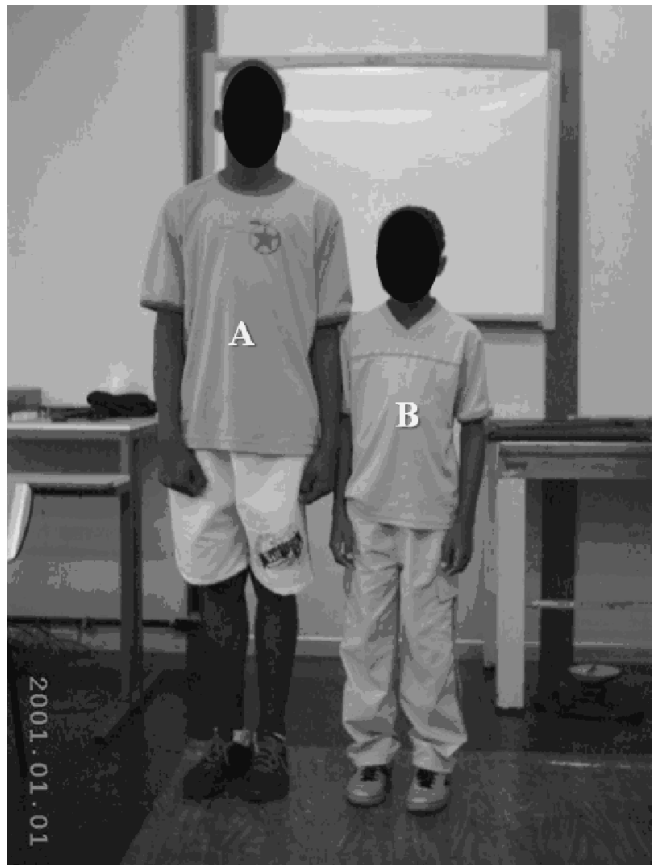

Figura 1. Voluntários avaliados no projeto de pesquisa LabCine/UERJ (2001)

Ambos com 12 anos de idade cronológica, sendo que o indivíduo A possui 13 anos de idade óssea, enquanto que o indivíduo $B$ possui apenas 9 anos de idade óssea. 
púbere - IPVC); c) percentagem da estatura em adulto atingida até um determinado momento; d) idade morfológica que se obtém ao justapor a estatura alcançada pelo jovem num determinado momento, com valores de referência das curvas de crescimento. Cabe salientar que estas alterações nas dimensões corporais podem sofrer grande variação individual, uma vez que crianças com uma mesma idade cronológica podem apresentar expressivas diferenças de tamanho corporal, refletindo de forma bastante significativa na estatura individual, como podemos observar na figura 1 .

\section{Limitações dos métodos}

Vários são os métodos disponíveis para avaliação da maturação biológica. Todavia, nem todos atendem às condições listadas por Guedes $\&$ Guedes $^{23}$ e por Beunen e colaboradores ${ }^{13}$ como fundamentais para sua validade, quais sejam: a) refletir mudanças em um sistema biológico; b) ocorrer em todos os indivíduos enquanto progridem para o estado adulto; c) alcançar o mesmo estágio final em todos os indivíduos; d) mostrar um progresso contínuo de tal forma que estágios discretos possam ser identificados em um continuum; e) aplicabilidade durante todo processo de maturação orgânica; f) ser independente do crescimento corporal. Isso se reflete, aliás, na quantidade de estudos que adotaram as diferentes abordagens apresentadas na determinação do estado de maturação.

Embora se acredite que a medida endócrina possa proporcionar uma avaliação mais direta das mudanças na puberdade, as exigências para a avaliação endócrina, a quantidade de hormônios envolvidos com a puberdade e os problemas práticos em obter medidas válidas são substanciais. ${ }^{21}$

A maturidade morfológica, embora bastante utilizada e de fácil aplicabilidade, ao considerar a estatura como indicador maturacional questiona a importância da genética na determinação da estatura adulta. Sua aplicabilidade fica restrita aos indivíduos cujas estaturas dos pais são conhecidas ou pode ser medida, inviabilizando- se para os demais indivíduos, a menos que seja determinada a idade óssea. No entanto, essa determinação por si só já seria suficiente para estimar a maturidade biológica.

Os métodos com foco na maturidade sexual restringem-se ao período peripuberal, determinando estágios discretos e descaracterizando o processo contínuo da maturação biológica. Além disso, existem questionamentos quanto à invasão da privacidade dos jovens em sua aplicação. A maturidade dental refere-se, especificamente, a um sistema biológico, além de ser dependente dos cuidados na manutenção e da possibilidade de extração dos dentes. Sua utilização é, portanto, sensivelmente mais restrita que as demais abordagens. Já a maturidade hormonal, tomada isoladamente, não consegue discriminar convenientemente os estágios de maturação. $\mathrm{Na}$ verdade, um determinado valor de concentração de testosterona (ou de outro hormônio de referência qualquer) pode estar relacionado a mais de um estágio de crescimento e desenvolvimento.

Embora se afirme que o método ideal de avaliação da maturação óssea deva incluir o estudo de todo o esqueleto, por razões práticas e econômicas a maturação do esqueleto está focada principalmente sobre os ossos da mão e punho, ${ }^{8}$ embora outros segmentos corporais, como vértebras cervicais, joelho, quadril e pé, também sejam utilizados. ${ }^{21}$

\section{Conclusão}

A maturidade óssea, apesar da pequena exposição à radiação a que se submete o avaliado, é o método que parece melhor atender às condições propostas, uma vez que reflete mudanças em um sistema biológico, ocorre em todos os indivíduos enquanto progridem para o estado adulto e alcança o mesmo estágio final (esqueleto ossificado) em todos os indivíduos. Além disso, mostra, através da idade óssea, um progresso contínuo de tal forma que estágios discretos podem ser identificados em um continuum, sendo aplicável durante todo 
processo de maturação orgânica de forma independente do crescimento corporal. Sendo assim, a maturidade esquelética, através da identificação da idade óssea, talvez se constitua no método que reúne mais vantagens na determinação do estágio maturacional de jovens, mesmo que estes estejam no período peripuberal.

\section{Referências}

1. Malina RM. Growth and maturation: basic principles and effects of training. In: Malina R, Silva MC, coord. Children and Youth in Organized Sports. Coimbra: Coimbra University Press; 2004. p. 137-61.

2. Malina RM, Bouchard C, Bar-Or O. Growth, maturation and physical activity. 2nd ed. Champaign, IL: Human Kinetics; 2004.728 p.

3. Bogin B. Patterns of Human Growth. 2nd ed. Cambridge: Cambridge University Press; 1999. $472 \mathrm{p}$.

4. Ellis BJ. Timing of pubertal maturation in girls: an integrated life history approach. Psychol Bull. 2004 Nov;130(6):920-58.

5. Cameron N. Assessment of Maturation. In: Cameron N, Bogin B, editors. Human Growth and Development. San Diego: Academic Press; 2002. p. 363-82.

6. Schubert CM, Chumlea WC, Kulin HE, Lee PA, Himes JH, Sun SS. Concordant and discordant sexual maturation among U.S. children in relation to body weight and BMI. J Adolesc Health. 2005 Nov;37(5):356-62.

7. de Muinich Keizer SM, Mul D. Trends in pubertal development in Europe. Hum Reprod Update. 2001 May-Jun;7(3):287-91.

8. Grave KC, Brown T. Skeletal ossification and the adolescent growth spurt. Am J Orthod. 1976 Jun;69(6):611-9.

9. Tanner JM. Growth at Adolescence. 2nd ed. Oxford: Blackwell Scientific Publications; c1962. Chapter 2, The development of the reproductive system; p. 28-39.

10. Hägg U, Taranger J. Maturation indicators and the pubertal growth spurt. Am J Orthod. 1982 Oct;82(4):299-309.

11. Eveleth PB, Tanner JM. Worldwide variation in human growth. 2nd ed. Cambridge: Cambridge University Press; 1990. 412 p.
12. Bond L, Clements J, Bertalli N, EvansWhipp T, McMorrisBJ, Patton GC et al. A comparison of self-reported puberty using the Pubertal Development Scale and the Sexual Maturation Scale in a school-based epidemiologic survey. J Adolesc. 2006 Oct;29(5):709-20.

13. Beunen GP, Rogol AD, Malina RM. Indicators of biological maturation and secular changes in biological maturation. Food Nutr Bull. 2006 Dec;27(4 Suppl Growth Standard):S244-56.

14. Duarte MFS. Maturação física: uma revisão da literatura, com especial atenção à criança brasileira. Cad Saúde Pública. 1993;9(supl 1):71-84.

15. Malina RM, Cumming SP, Morano PJ, Barron M, Miller SJ. Maturity status of youth football players: a noninvasive estimate. Med Sci Sports Exerc. 2005 Jun;37(6):1044-52.

16. Malina RM, Bouchard C, Bar-Or O. Growth, maturation and physical activity. 2nd ed. Champaign, IL: Human Kinetics; c2004. Chapter 15, Biological maturation: concepts and assessment; p. 277-306.

17. Gertych A, Zhang A, Sayre J, PospiechKurkowska S, Huang HK. Bone age assessment of children using a digital hand atlas. Comput Med Imaging Graph. 2007 JunJul;31(4-5):322-31.

18. Tanner JM, Healy MJR, Goldstein H, Cameron N. Assessment of Skeletal Maturity and Prediction of Adult Height (TW3 Method). 3rd ed. London: W. B. Saunders; 2001. 128 p.

19. Baccetti T, Franchi L, De Lisa S, Giuntini V. Eruption of the maxillary canines in relation to skeletal maturity. Am J Orthod Dentofacial Orthop. 2008 May;133(5):748-51.

20. Demirjian A, Goldstein H, Tanner JM. A new system of dental age assessment. Hum Biol. 1973 May;45(2):211-27.

21. Petersen AC, Crockett L, Richards M, Boxer A. A self-report measure of pubertal status: Reliability, validity, and initial norms. J Youth Adolesc. 1988;17(2):117-33.

22. Fragoso I, Vieira F. Morfologia e Crescimento. Cruz Quebrada: Faculdade de Motricidade Humana; 2000. 287 p.

23. Guedes DP, Guedes JERP. Crescimento, Composição Corporal e Desempenho Motor de Crianças e Adolescentes. São Paulo: CLR Balieiro; 1997. 362 p. 


\section{Autores}

\section{Ada Fernanda P. S. Lima}

Laboratório de Atividade Física e Promoção da Saúde. Instituto de Educação Física e Desportos. Universidade do Estado do Rio de Janeiro. Rio de Janeiro, RJ, Brasil.

\section{Alexandre H. Okano}

Departamento de Educação Física. Centro de Ciências da Saúde. Universidade Federal do Rio Grande do Norte. Natal, RN, Brasil.

\section{Alice R. Sampaio}

Laboratório de Atividade Física e Promoção da Saúde. Instituto de Educação Física e Desportos. Universidade do Estado do Rio de Janeiro. Rio de Janeiro, RJ, Brasil.

\section{Ana Paula M. Guttierres}

Laboratório de Atividade Física e Promoção da Saúde. Instituto de Educação Física e Desportos. Universidade do Estado do Rio de Janeiro. Rio de Janeiro, RJ, Brasil.

\section{Astrogildo V. Oliveira Júnior}

Departamento de Educação Física e Folclore. Colégio Pedro II. Rio de Janeiro, RJ, Brasil.

\section{Brenno S. Silva}

Programa de Pós-graduação em Ciências da Atividade Física. Universidade Salgado de Oliveira. Niterói, RJ, Brasil.

\section{Daniel A. Bottino}

Laboratório de Pesquisas Clínicas e Experimentais em Biologia Vascular. Centro Biomédico.

Universidade do Estado do Rio de Janeiro. Rio de Janeiro, RJ, Brasil.

\section{Dionizio Mendes Ramos Filho}

Laboratório de Bioenergética e Fisiologia Mitocondrial. Centro de Ciências da Saúde. Universidade Federal do Rio de Janeiro. Rio de Janeiro, RJ, Brasil.

\section{Elirez B. Silva}

Laboratório de Pesquisa Clínica Escola. Departamento de Fisioterapia. Universidade Gama Filho. Rio de Janeiro, RJ, Brasil.

\section{Ercole C. Rubini}

Laboratório de Fisiologia do Exercício. Universidade Estácio de Sá. Rio de Janeiro, RJ, Brasil.

\section{Fabrício V. A. Vasconcellos}

Centro de Investigação, Formação, Inovação, Intervenção e Desporto. Faculdade de Desporto. Universidade do Porto. Porto, Portugal.

\section{Felipe A. da Cunha}

Programa de Pós-graduação em Ciências Médicas. Universidade do Estado do Rio de Janeiro. Rio de Janeiro, RJ, Brasil.

\section{Fernanda Monteiro}

Laboratório de Atividade Física e Promoção da Saúde. Instituto de Educação Física e Desportos. Universidade do Estado do Rio de Janeiro. Rio de Janeiro, RJ, Brasil.

\section{Flávia Porto}

Programa de Pós-graduação em Ciências do Exercício e do Esporte. Universidade Gama Filho. Rio de Janeiro, RJ, Brasil. 


\section{Gustavo C. Lopes}

Laboratório de Atividade Física e Promoção da Saúde. Instituto de Educação Física e Desportos. Universidade do Estado do Rio de Janeiro. Rio de Janeiro, RJ, Brasil.

\section{Jonas L. Gurgel}

Programa de Pós-graduação em Ciências

Cardiovasculares. Universidade Federal Fluminense.

Niterói, RJ, Brasil.

\section{Jonathan Myers}

VA Palo Alto Health Care System. Cardiology Division. Stanford University. Palo Alto, California, United States.

\section{Karynne Grutter}

Laboratório de Anatomia Humana. Universidade Castelo Branco. Rio de Janeiro, RJ, Brasil.

\section{Lenifran Matos-Santos}

Programa de Pós-graduação em Ciências da Atividade Física. Universidade Salgado de Oliveira. Niterói, RJ, Brasil.

\section{Lorena Paes}

Programa de Pós-graduação em Fisiopatologia Clínica e Experimental. Universidade do Estado do Rio de Janeiro. Rio de Janeiro, RJ, Brasil.

\section{Luciane P. da Costa}

Laboratório de Atividade Física e Promoção da Saúde. Instituto de Educação Física e Desportos. Universidade do Estado do Rio de Janeiro. Rio de Janeiro, RJ, Brasil.

\section{Luiz G. Kraemer-Aguiar}

Departamento de Medicina Interna. Faculdade de Ciências Médicas. Universidade do Estado do Rio de Janeiro. Rio de Janeiro, RJ, Brasil.

\section{Matheus R. Hausen}

Laboratório de Atividade Física e Promoção da Saúde. Instituto de Educação Física e Desportos. Universidade do Estado do Rio de Janeiro. Rio de Janeiro, RJ, Brasil.

\section{Paulo T. V. Farinatti}

Laboratório de Atividade Física e Promoção da Saúde. Instituto de Educação Física e Desportos. Universidade do Estado do Rio de Janeiro. Rio de Janeiro, RJ, Brasil.

\section{Rafael A. Montenegro}

Programa de Pós-graduação em Fisiopatologia Clínica e Experimental. Universidade do Estado do Rio de Janeiro. Rio de Janeiro, RJ, Brasil.

\section{Raul A. Freire}

Programa de Pós-graduação em Ciências da Atividade Física. Universidade Salgado de Oliveira. Niterói, RJ, Brasil.

\section{Renato O. Massaferri}

Programa de Pós-graduação em Ciências da Atividade Física. Universidade Salgado de Oliveira. Niterói, RJ, Brasil.

\section{Ricardo B. Oliveira}

Laboratório de Atividade Física e Promoção da Saúde. Instituto de Educação Física e Desportos. Universidade do Estado do Rio de Janeiro. Rio de Janeiro, RJ, Brasil.

\section{Ricardo G. Cordeiro}

Programa de Pós-graduação em Ciências da Atividade Física. Universidade Salgado de Oliveira. Niterói, RJ, Brasil.

\section{Sérgio Machado}

Programa de Pós-graduação em Ciências da Atividade Física. Universidade Salgado de Oliveira. Niterói, RJ, Brasil.

\section{Taciana Pinto}

Laboratório de Atividade Física e Promoção da Saúde. Instituto de Educação Física e Desportos. Universidade do Estado do Rio de Janeiro. Rio de Janeiro, RJ, Brasil.

\section{Tainah P. Lima Monteiro}

Programa de Pós-graduação em Ciências Médicas. Faculdade de Ciências Médicas. Universidade do Estado do Rio de Janeiro. Rio de Janeiro, RJ, Brasil.

\section{Tânia M. P. F. Paschoalino}

Hospital Universitário Antônio Pedro. Universidade Federal Fluminense. Niterói, RJ, Brasil.

\section{Walace D. Monteiro}

Laboratório de Atividade Física e Promoção da Saúde. Instituto de Educação Física e Desportos. Universidade do Estado do Rio de Janeiro. Rio de Janeiro, RJ, Brasil. 